\title{
Can $p$-values be meaningfully interpreted without random sampling?
}

Abstract: Besides the inferential errors that abound in the interpretation of $p$-values, the probabilistic pre-conditions (i.e. random sampling or equivalent) for using them at all are not often met by observational studies in the social sciences. This paper systematizes different sampling designs and discusses the restrictive requirements of data collection that are the sine-qua-non for using $p$-values.

Keywords: sampling design, propensity scores, $p$-values, random sampling, selection bias, inference JEL: C10, C83

\section{Contributing authors}

Prof. Dr. Norbert Hirschauer (Corresponding Author)

Martin Luther University Halle-Wittenberg, Faculty of Natural Sciences III

Institute of Agricultural and Nutritional Sciences, Chair of Agribusiness Management

Karl-Freiherr-von-Fritsch-Str. 4, D-06120 Halle (Saale)

norbert.hirschauer@landw.uni-halle.de

\section{Dr. Sven Grüner}

Martin Luther University Halle-Wittenberg, Faculty of Natural Sciences III

Institute of Agricultural and Nutritional Sciences, Chair of Agribusiness Management

Karl-Freiherr-von-Fritsch-Str. 4, D-06120 Halle (Saale)

sven.gruener@landw.uni-halle.de

\section{Prof. Dr. Oliver Mußhoff}

Georg August University Goettingen

Department for Agricultural Economics and Rural Development, Farm Management

Platz der Göttinger Sieben 5, D-37073 Göttingen

Oliver.Musshoff@agr.uni-goettingen.de

\section{Prof. Dr. Claudia Becker}

Martin Luther University Halle-Wittenberg, Faculty of Law and Economics

Institute of Business Studies, Chair of Statistics

Große Steinstraße 73, D-06099 Halle (Saale)

claudia.becker@wiwi.uni-halle.de

\section{Antje Jantsch}

Leibniz Institute of Agricultural Development in Transition Economies (IAMO)

External Environment for Agriculture and Policy Analysis (Agricultural Policy)

Theodor-Lieser-Str. 2, D-06120 Halle (Saale)

jantsch@iamo.de 


\section{Can $p$-values be meaningfully interpreted without random sampling?}

Abstract: Besides the inferential errors that abound in the interpretation of $p$-values, the probabilistic pre-conditions (i.e. random sampling or equivalent) for using them at all are not often met by observational studies in the social sciences. This paper systematizes different sampling designs and discusses the restrictive requirements of data collection that are the sine-qua-non for using $p$-values.

Keywords: sampling design, propensity scores, $p$-values, random sampling, selection bias, inference

JEL: C10, C83

\section{Introduction}

Statistical inference in the study of observational data is concerned with random (sampling) error, i.e. the fact that even a random sample does not exactly reflect the properties of the population and that there is often considerable sample-to-sample variation when we repeatedly draw random samples from a population. Inferential tools based on standard errors, such as $p$-values and confidence intervals, are to help make inductive inferences beyond the confines of a particular sample to a population. Due to their probabilistic foundation, they require a random process of data generation that can hypothetically be replicated. When $p$-values are displayed, a plausible argument should be given that the studied sample meets the underlying probabilistic assumptions, i.e. that it is a random sample or that the deviation from random sampling can be ignored or has been corrected for. Otherwise, there are no grounds for using $p$-values and they become essentially uninterpretable (COPAS and LI 1997; VOGT et al. 2014). In general, researchers should transparently describe how and from which parent population the sample was drawn and, consequently, to which inferential (target) population they want to generalize (HIRSCHAUER et al. 2019a). ${ }^{1}$

In the economic and social sciences, we rarely have random samples. We often use pre-existing observational data or survey data obtained from convenience samples that are non-random but easy to come by at low costs. But even if we take the trouble of, and succeed in, randomly selecting subject pools from defined populations, we usually do not escape sample selection bias because we cannot force subjects to participate. Due to self-selection, participants and non-participants in a survey might be systematically different. ${ }^{2}$ The problem of non-probabilistically sampled data can be framed as a missing data problem where data are not missing at random. As a consequence, estimates of population quantities including regression coefficients as well as the standard errors and $p$-values of those estimates might be biased in unknown ways. Non-random sample selection is often obvious. Nonetheless, many studies tacitly proceed as if they had a random sample and follow a misguided routine of always displaying $p$ values for regression coefficients. Resulting conclusions run the risk of being grossly misleading.

Simple random sampling clearly represents a chance-based sampling design that allows for a meaningful interpretation of $p$-values. ${ }^{3}$ When deviating from this ideal, things become less obvious and it is often unclear under which conditions $p$-values can be used as an inferential aid at all. Five ideal-type sampling contexts can be distinguished regarding the use of $p$-values: (i) simple random sampling, (ii) stratified

\footnotetext{
${ }^{1}$ This paper focuses on inductive statistical inference in terms of estimating population quantities and generalizing from a sample to its parent population. The fact, however, that a probabilistic data generation mechanism is the sine-qua-non for a meaningful interpretation of $p$-values also holds for experimental approaches that are aimed at identifying causal effects within a given sample of study (cf. HIRSCHAUER et al. 2019b).

${ }^{2}$ In longitudinal studies, we face the additional problem of attrition when study participants "get lost" over time.

${ }^{3}$ Even so, one should be cautious. Over the last decades, a vast literature has evolved warning against inferential errors associated with $p$-values. A review of this literature is beyond the scope of this paper. For first insights see ZILIAK and MCCLOSKEY (2008), KRÄMER (2011), GIGERENZER and MAREWSKI (2015), GREENLAND et al. (2016), BERRY (2017), GELMAN and CARLIN (2017), TrAFimOV et al. (2018), Hirschauer et al. (2018, 2019a). The topicality of this issue is reflected in the unprecedented 2016 -value warning of the American Statistical Association (WASSERSTEIN and LAZAR 2016), its $p$-value symposium in fall 2017, the $p$-value Special Issue on "Statistical inference in the 21 st century: A world beyond P < 0.05 " of The American Statistician (WASSERSTEIN et al. 2019), and the $p$-value petition to retire statistical significance in Nature (AMRHEIN et al. 2019).
} 
sampling, (iii) cluster sampling, (iv) sampling contaminated with selection bias, and (v) full population surveys. ${ }^{4}$ All of them are discussed in this paper to facilitate the understanding of the probabilistic preconditions for using $p$-values and the fact that they are only meaningful when these conditions are met.

\section{Sampling designs that facilitate the use of inferential statistics}

\subsection{Simple random sampling - the generic benchmark for statistical inference}

To understand the purpose of statistical inference, one must clearly distinguish between sample quantities (estimates) and population quantities. Otherwise one runs the risk of lurching all-too readily from the description of some sample data that are conveniently at hand to overconfident generalizations regarding population quantities (MATTHEWS et al. 2017). Failing to be explicit regarding the data generation process and the inferential target population fuels the risk of rash statements regarding real-world regularities. BERK and FREEDMAN (2003) emphasize that statistical assumptions are empirical commitments and that acting as if one obtained data through random sampling does not create a random sample. Complying with the empirical procedure "random sampling" permits using $p$-values as inferential aids for inductive generalizations from the probability sample towards its parent population. Non-compliance, in contrast, precludes a meaningful use of inferential statistics, except when deviations from random-sampling are demonstrably ignorable or adequately corrected for. The most basic case of a probability sample is a simple random sample (SRS), i.e. a subset of a population drawn with the same probability as any other conceivable subset of identical size, such that each unit had equal probability of being selected into the subset. Being an unbiased representation of the population, a SRS permits not only the use of inferential statistics such as $p$-values but also the use of conventional estimates such as the sample mean as unbiased estimate of the population mean.

Despite delusive significance declarations that are often attached to $p$-values, their inferential content is limited even when they are applicable in principle. In the words of MCCLOSKEY and ZILIAK (1996: 98) we must realize that " $[\mathrm{t}] \mathrm{h}$ uncertainty of the estimate that arises from sampling error [is] the only kind of uncertainty the test of significance deals with." In other words, the $p$-value compares a point estimate from a particular random sample with an estimated noise-only distribution of outcomes that would result if random error were at work alone (LUDWIG 2005). This distribution is inherently based on the thought experiment of statistical replication, i.e. we assume noise-only and presumably apply the same econometric model to many other equal-sized random samples from the same population. In brief, the $p$-value is the conditional probability of re-finding an observed effect (or a larger one) in random replications if we assumed the null hypothesis of zero effect to be true. Per definition, a $p$-value cannot work inversely and inform us on the underlying reality; i.e. "it is logically impossible to infer from the $p$-value whether the null hypothesis or an alternative proposition is true. We cannot even derive probabilities for hypotheses based on what has delusively become known as 'hypothesis testing.' In the usual sense of the word, a $p$-value cannot 'test' or 'confirm' a hypothesis, but only describe data frequencies under a certain statistical model including the null” (HIRSCHAUER et al. 2019a: 712).

\subsection{Stratified sampling}

In many surveys, researchers deviate from simple random draws of the population because other sampling designs are statistically or financially more efficient. In stratified sampling, for example, we first divide a population of size $N$ into $H$ mutually heterogeneous but internally as homogeneous as possible subpopulations ("strata"). We then randomly sample from each stratum $h(h \in\{1,2, \ldots, H\})$ independently. The simplest case is proportionate stratified sampling where we sample an identical fraction

\footnotetext{
${ }^{4}$ There is a wide variety of other and more complex and mixed sampling designs. While their respective features need to be considered to obtain unbiased estimates of population quantities and standard errors, it is beyond this paper's scope to go into the details of these designs (cf. e.g. LOHR 2009).
} 
of each stratum $\left(n_{1} / N_{1}=n_{2} / N_{2}=\ldots=n_{H} / N_{H}\right)$, ensuring that the stratum sample size $n_{h}$ is proportional to stratum size $N_{h}$. Disproportionate stratified sampling, in contrast, intentionally oversamples certain strata - for example those that exhibit more variability than others - to reduce sampling error.

Contrary to proportionate stratified sampling, where each unit has equal probability of being included into the sample, we need to consider differential weights when a sample is systematically unrepresentative of the population such as in disproportionate stratified sampling (SOLON et al. 2013: 5). In other words, we need to "reconfigure the sample as if it was a simple random draw of the total population" (FRIEDMAN 2013). The weight $w_{h i}=w_{h}=N_{h} / n_{h}$ that is assigned to a sampled unit $i$ ( $i \in$ $\left.\left\{1,2, \ldots, n_{h}\right\}\right)$ in stratum $h$ is the reciprocal of the probability that this unit is included in the sample. It indicates how many units of the full population are represented by a sampled unit. If we sample a 10\%fraction in stratum 1 and a 20\%-fraction in stratum 2, then each sampled unit in stratum 1 has weight $w_{1}=10$ (represents 10 units), whereas each sampled unit in stratum 2 has weight $w_{2}=5$ (represents 5 units). When estimating a population regression from such a sample, we need to apply, in the simplest case, weighted least squares instead of ordinary least squares to the sample regression to obtain unbiased point estimates (SOLON et al. 2013).

Stratified sampling needs also to be considered when estimating sampling variability. Compared to a SRS of size $n$, stratified sampling, where $n=\sum n_{h}$, reduces sampling error. The estimated standard error of the mean, for example, is given by (cf. LOHR 2009: 79): ${ }^{5}$

$\widehat{S E}_{\text {Strat }}=\left(\sum \frac{N_{h}^{2}}{N^{2}} \cdot \frac{N_{h}-n_{h}}{N_{h}} \cdot \frac{s_{h}^{2}}{n_{h}}\right)^{0.5}$,

where $s_{h}^{2}$ is the sample variance within each stratum $h$.

When estimating the standard error for stratified samples, we consider the within-strata variance but not the variance between strata because we independently draw a random sample from each stratum. This is why, compared to simple random sampling, the reduction of the standard error is the more pronounced the smaller the variance within the strata and the greater the variance between them. Equation (1) also uses a finite population correction $\left(N_{h}-n_{h}\right) / N_{h}=1-n_{h} / N_{h}$. The correction considers that sampling error not only decreases with growing sample size but also when sample size becomes large relative to the population (HIRSCHAUER et al. 2019b). Since the correction applies within each stratum, it can be used to make stratified sampling still more efficient: it reduces the standard error when strata with high variability are oversampled. Such oversampling can also be seen as an intentional use of low weights for strata with high variability. Using $w_{h}=N_{h} / n_{h}$, this can be shown by rewriting equation (1) as:

$\widehat{S E}_{\text {Strat }}=\frac{1}{N} \cdot\left(\sum N_{h} \cdot\left(N_{h}-n_{h}\right) \cdot \frac{s_{h}^{2}}{n_{h}}\right)^{0.5}=\frac{1}{N} \cdot\left(\sum w_{h} \cdot\left(N_{h}-n_{h}\right) \cdot s_{h}^{2}\right)^{0.5}$

We may summarize that stratified sampling is a special case of random sampling. Not only are the probabilistic pre-conditions (i.e. known selection probabilities) for using standard errors and $p$-values met, but standard errors and $p$-values can be adjusted downwards. If we neglected this downward adjustment and used the conventional standard error for a SRS, we would be too conservative in our judgement - insofar as we find it useful to resort to standard errors and $p$-values as inferential aids at all.

\subsection{Cluster sampling}

Cluster sampling (e.g. area sampling) is used in practice because randomly sampling from preselected segments of the population ("clusters") is usually much cheaper and more convenient than randomly sampling from the full population (LOHR 2009: 167). There is a superficial similarity of cluster sampling

\footnotetext{
${ }^{5}$ Formal representations throughout this paper focus on the mean because the notation for the estimation of standard errors of other quantities such as regression coefficients tends to become messy. The universal argument, however, that standard errors generally presuppose a random process of data generation and that deviations from simple random sampling need to be considered when estimating standard errors applies to all estimates.
} 
to stratified sampling because both subdivide a population of size $N$ into exhaustive subpopulations (segments). However, earmarking the fundamental difference in the sampling design, we use $G$ to denote the number of segments that are now called "clusters." The difference to stratified sampling is that, instead of randomly drawing observational units from each segment of the population, we now adopt a hierarchical approach to data collection: in a primary step, we draw a random sample of $g$ clusters from the population of $G$ clusters, which are therefore called "primary sampling units" (psus). In a second step, we randomly select observational units, which are called "secondary sampling units" (ssus), from the previously selected clusters. Both stages of data collection need to be accounted for when estimating population quantities and standard errors. LOHR (2009: 168) warns that "[o]ne of the biggest mistakes made by researchers using survey data is to analyze a cluster sample as if it were an SRS. Such confusion usually results in the researchers reporting standard errors that are much smaller than they should be."

A simple variant of cluster sampling is one-stage cluster sampling where all observational units (ssus) within the selected clusters (psus) are surveyed. When this is too costly, two-stage cluster sampling is applied; i.e. instead of fully surveying selected clusters, we draw a SRS of units from each selected cluster. Analogous to stratified sampling, we need to consider differential weights when estimating population quantities from cluster samples that are systematically unrepresentative of the population. The appropriate weight is still the reciprocal of a unit's probability of being included into the sample. However, this probability now derives from the probability that a cluster is selected in the first stage and the (conditional) probability that a unit within a selected cluster is sampled in the second stage.

Whereas stratification decreases sampling error compared to a SRS, the opposite is generally true for cluster sampling. LOHR (2009: 166) illustrates why: "Members of the same cluster tend to be more similar than elements selected at random from the whole population - $[\ldots]$; residents of the same nursing home tend to have similar opinions of the quality of care. These similarities usually arise because of some underlying factors that may or may not be measurable- $-[. .$.$] . Thus, we do not obtain as much$ information about all nursing home residents in the United States by sampling two residents in the same home as by sampling two residents in different homes, [...]." cluster sampling occurs even when we use equal selection probabilities for all ssus that facilitate a conventional ("self-weighting") calculation of unbiased point estimates analogous to a SRS. ${ }^{7}$

In one-stage clustering with clusters of equal size $N / G$ that are selected with equal probability, estimating the standard error of the mean is straightforward (cf. LOHR 2009: 171):

$\widehat{S E}_{\text {Clust_1 }}=1 / \frac{N}{G} \cdot\left(\left(1-\frac{g}{G}\right) \cdot \frac{s_{t}^{2}}{g}\right)^{0.5}$,

where $s_{t}^{2}$ is the between-psu variance of cluster totals.

\footnotetext{
${ }^{6}$ The literature cautiously states that cluster sampling "generally" increases standard errors. This is because units in natural clusters such as geographical areas often share environmental influences that make them more homogeneous than units that are randomly selected from the full population. Measuring this homogeneity, we would find that cluster members exhibit positive intra-cluster correlations. The opposite can be imagined in principle; i.e. the units within a cluster could be less homogeneous than randomly selected units and exhibit negative intra-cluster correlations. While this is unlikely in natural clusters, it might occur in artificial clusters (LOHR 2009: 173ff).

${ }^{7}$ There are two approaches of obtaining equal probabilities for all observational units (ssus): (i) we use probabilities proportional to cluster size when selecting clusters (psus) and then sample the same absolute number of ssus in each psu, or (ii) we use a fixed probability for selecting psus and then sample an identical fraction of ssus in each psu (one-stage clustering is a special case of this approach). Imagine a population of size 1,000 that is divided into 5 large psus à 100 and 10 small psus à 50. Approach (i) implies that sampling a large psu is twice as likely as sampling a small psu (e.g. 0.2 vs. 0.1). An equal number of ssus (e.g. 25), is then sampled in each psu - corresponding to a fraction of 0.25 in large psus and a fraction of 0.5 in small psus. The resulting probability of ssus being selected would be 0.05 , both for ssus in large psus $(0.05=0.2 \cdot 0.25)$ and for ssus in small psus $(0.05=$ $0.1 \cdot 0.5)$. In approach (ii), an equal weight could be obtained, for example, by using an equal probability of 0.1 for selecting psus and sampling an identical 50\%-fraction of ssus within each psu. While both approaches are selfweighting, approach (ii) is expected to produce an even larger sampling error than approach (i) (LOHR 2009: 220).
} 
Equation (2) shows that in one-stage clustering, the standard error depends on the between-psu variance but not the within-psu variance. Even when we maintain the assumption of equal-sized clusters that are selected with equal-probability, the standard error formula becomes complex in two-stage clustering: we now need to consider the between-psu and the within-psu variance because sampling error results from two sources: from sampling $g$ clusters in the first stage and from sampling $n / g$ units within each selected cluster in the second stage (cf. LOHR 2009: 185):

$\widehat{S E}_{\text {Clust_2 }}=1 / \frac{N}{G} \cdot\left(\left(1-\frac{g}{G}\right) \cdot \frac{s_{t}^{2}}{g}+\frac{1}{g^{2}} \sum_{i \in \text { sampled }} \frac{N^{2}}{G^{2}} \cdot\left(1-\frac{n / g}{N / G}\right) \cdot \frac{s_{i}^{2}}{n / g}\right)^{0.5}$,

where $s_{i}^{2}$ is the within-psu variance of sampled observations from the ith sampled cluster, and $n$ is the total sample size. ${ }^{8}$

If we analysed the data as if they were obtained from a simple random draw of the full population, we would mistakenly estimate the standard error as $\widehat{S E}_{S R S}=\left((1-n / N) \cdot s^{2} / n\right)^{0.5}$, with $s^{2}$ being the variance of all observations in a sample of size $n$, and $1-n / N$ being the finite population correction. ${ }^{9,10,11}$

We know that basing the calculation of standard errors in a cluster sample - be it for a mean or a regression coefficient - on the SRS assumption generally leads to an underestimation. This underestimation is sometimes quantified by the so-called "design effect," which is the ratio of the sampling variance that results from the cluster sampling design to the sampling variance of a SRS of the same size (KISH 1965: 161). The design effect tells us by how much we would underestimate the variance if we erroneously used the formula for a SRS of the same $n$, but "it is not a way to avoid calculating variances: You need an estimate of the variance from the complex design to find the design effect" (LOHR 2009: 309). CAMERON and MILLER (2015: 2) note that "[i]t is not unusual to have applications where standard errors that control for within cluster correlations are several times larger than the default standard errors that ignore such correlation."

We may summarize that cluster sampling is another special case of random sampling where the probabilistic pre-conditions for using standard errors and $p$-values are met. However, cluster samples usually produce larger sampling errors than SRSs. Hence, standard errors and $p$-values need to be adjusted upwards. Otherwise we would be too confident and, even worse, too confident to an unknown degree, in our inferential judgements - insofar as they are based on standard errors and $p$-values in the first place.

\footnotetext{
${ }^{8}$ When all clusters are selected in the first stage $(g=G)$, we, again, have stratified sampling. Aligning the notation ( $g=G=H, N / G=N_{h}, n / g=n_{h}, s_{i}^{2}=s_{h}^{2}$ ), equation (3) can correspondingly be reduced to equation (1).

${ }^{9}$ Even in a SRS design, we need to use "heteroscedasticity-robust" standard errors when the dispersion of observations is different in different segments of the population.

${ }^{10}$ It is essential to consider the ex-ante known features of the sampling design. When data were collected by others, researchers might ignore the particular design. While it is common to use cluster-robust standard errors in such circumstances, it might be flawed. Let's assume a researcher who ignores that data resulted from stratified sampling and finds that observations are similar in certain segments of the population (e.g. provinces). These provinces might then be considered as clusters and a cluster-robust estimation based on the usual assumption that the total number of clusters goes to infinity would find standard errors that are larger than conventional standard errors. They should be smaller, however, because in the stratified sampling design each province was selected.

${ }^{11}$ Even when we know that we have a SRS design, an ex-post data analysis might reveal that there are intra-cluster correlations. The literature on cluster-robust estimation focuses on this "ex-post identification of clusters." For an introduction see CAMERON and TRIVEDI (2005, 2009), CAMERON and MILLER (2015) or MACKINNON (2019) and the literature referenced therein. This literature is first of all based on the assumption that the total number of clusters is large. CAMERON and MILLER (2015: 23) note that, when this is not the case, even cluster-robust standard errors can be downwards-biased. At the same time, the number of observed clusters should be small relative to the population of clusters. Noting that many economic datasets do not meet this condition, ABADIE et al. (2017) find that cluster-robust standard errors are often upwards-biased. This raises the question of how cluster-robust standard errors are to be estimated in the SRS case when there is a small population of clusters (e.g. 20 geographical areas) and a number of observed clusters (e.g. 10) that is not small relative to the population (cf. CAMERON and MILLER 2015).
} 


\section{Sampling designs that preclude the use of inferential statistics}

\subsection{Convenience samples contaminated by bias from non-coverage and self-selection}

Observational data are often tacitly analyzed as if they were obtained through random sampling even when a non-random selection mechanism was at work. Such approaches can be seriously flawed. TRAFIMOW (2019: 344) unmistakably warns that "it is the rare study that uses sampling that is completely random and where each participant sampled is independent of the other participants sampled. Without making assertions about exactly how often these assumptions are importantly violated, there can be little doubt that the violation prevalence is considerable. In that case, no inferential statistical procedures, [...], are completely valid. [...] The notion that inferential statistical procedures may sometimes, and even often, be inappropriate, may be tough medicine for reviewers and editors to swallow. But the medicine nevertheless is therapeutic. Another option is to use methods for addressing violations of independence or random sampling [...], while keeping in mind that these have their own assumptions and drawbacks."

Using data from non-probability convenience samples is common in econometric studies (MILLER 2017). Non-coverage, i.e. when certain segments of the population are omitted from the sampling frame, is one instance that leads to a non-probabilistic sample composition. But even when we succeed in avoiding non-coverage and obtaining a sampling frame that contains a random pool of subjects, we usually do not escape self-selection because people can freely decide whether to respond to a survey or not. Selection problems can arise in all sampling designs and need to be accounted for in addition to the design-specific estimation procedures described in section 2. Because (non-)participation is rarely random (i.e. data are missing but not missing at random), the characteristics of respondents and non-respondents may be systematically different (selection bias). Therefore, sample quantities may tell us little about the population quantities of interest. In regression analysis, we run the risk of misestimating coefficients and standard errors in unknown ways unless we adequately correct for the selection bias that results from the violation of independent random sampling (cf. ROSENTHAL and ROSNOW 2009).

What we do in the attempt to correct for selection bias is best understood when looking back to stratified and cluster sampling. In both sampling designs, we knew ex ante the selection probabilities and the ways in which the data generating process was systematically different from a simple random draw of the full population. We therefore also knew how to "reconfigure" the sample to make it comparable to a simple random draw and ensure that the variable distribution in the sample matches the distribution in the parent population on average. When the composition of the sample is influenced by self-selection, we do not know these probabilities ex ante. We only know that (certain types of) people might select themselves into the sample with differing probabilities, depending on individual characteristics (age, sex, education, income etc.) that may be observable or not. Sample selection models are used to ex post control for these selection probabilities. They are based on the assumption that we know all confounding variables that might play a role in subjects' decisions to respond to a survey or not and that we have observations for these variables, both from respondents and non-respondents (no unmeasured confounding).

There is a wide variety of sample selection models. ${ }^{12}$ Among them, propensity score models are probably the most intuitive way to address selection bias. Propensity score models originated in (quasi-)experimental research focused on causal inferences within the group of experimental subjects (internal

\footnotetext{
${ }^{12}$ From the late 1970s, an extensive literature developed that concerned itself with selection problems. Two important research contexts were addressed: (i) experimental research where the identification of causal relationships is compromised when randomization is not fully successful (e.g. due to drop outs after randomization or quasiexperimental designs), and (ii) survey research where the unbiased estimation of population quantities is impaired when random sampling is not warranted (e.g. due to non-response). In the last two decades, a particular focus was put on selection problems in web surveys and, methodologically, on propensity score methods, which were imported to the survey context from experimental research. A review of the vast literature on sample selection is
} 
validity). In this context, they were used to deal with the problem of unbalanced confounders across treatment groups. ${ }^{13}$ Ideally, propensity score models use all selection-relevant variables to estimate subjects' probability (the "propensity score") of participation as opposed to non-participation (HECKMAN et al. 1997). Similar to the ex-ante known selection probabilities in stratified and cluster sampling, these ex-post estimated selection probabilities are then used to reconfigure the sample by correcting for any imbalances between those who are in the final sample and those who are not. Propensity scores are therefore also called "balancing scores," conditional on which the distribution of confounders in the response-group is assumed to match the distribution in the non-response group (AUSTIN 2011). ${ }^{14}$

Let us look at a stylized example to illustrate how propensity score methods work in principal: we are interested in the mean of a target variable $y$ (e.g. income) in a population with $N=10,000$ subjects. We presumable constructed a sampling frame that comprises 1,000 subjects (500 males and $500 \mathrm{fe}-$ males) and addressed a survey to all subjects on this sampling frame. The response rate was only $15 \%$, resulting in a sample size of $n=150$. In this sample, we find $n_{m}=100$ males (a 20\%-share of the male population on the sampling frame), but only $n_{f}=50$ females (a $10 \%$-share of the female population on the sampling frame). Assuming that gender is the only variable that affects selection (this is a critical assumption that we make for the sake of simplicity), we would equate the two gender shares with selection probabilities (propensity scores). That is, we would act on the assumption that males selected themselves into the sample with propensity $0.2(=100 / 500)$, whereas females did so with propensity $0.1(=50 / 500)$. To put it the other way round, each male in the sample has a weight $w_{m}=$ 5 (represents five males on the sampling frame) whereas each female has a weight $w_{f}=10$ (represents ten females on the sampling frame). Let's further assume that the mean among the 100 sampled males is $\bar{y}_{m}=7$, whereas the mean among the 50 sampled females is $\bar{y}_{f}=4$. This corresponds to a conventional sample mean $\bar{y}=6=(100 \cdot 7+50 \cdot 4) / 150)$. To correct for the fact that gender is not equally distributed across participants and non-participants (oversampling of males, undersampling of females), we resort to the weights derived from the propensity scores. Doing so, we obtain an estimate for the population mean of $\hat{\bar{y}}=5.5=(5 \cdot 100 \cdot 7+10 \cdot 50 \cdot 4) / 1,000$ (weights-corrected sample mean).

This highly stylized example corresponds to post-stratification; i.e. we use the selection-relevant characteristic "gender" to define population strata (i.e. a "male stratum" and a "female stratum") and then put the differing self-selection probabilities of males and females, which are beyond our control, on a level with intentional selection probabilities that we might have used in a disproportionate stratified sampling design. Hence, the same formulas as in the stratification case apply and equation (1) can be used to estimate the standard error of the mean in our stylized example (cf. DE LEEUW et al. 2008 for the estimation of standard errors under various versions of weight corrections). Our example was simple because gender was the only variable affecting selection and because there was one selection probability for all males and one selection probability for all females on the sampling frame. Compared to that,

beyond this paper's scope (see e.g. HECKMAN 1976, 1979; GREENE 1981; BERK 1983; WINSHIP and MARE 1992; HECKMAN et al. 1997; STOLZENBERG and RELLES 1997; VELLA and VERBEEK 1999; LEE and MARSH 2000; SHADISH et al. 2002; KALTON and FLORES-CERVANTES 2003; LUELLEN et al. 2005; GUO and FRASER 2009; ROSENBAUM 2010; AUSTIN 2011; BRICK 2013; VALLIANT et al. 2013; IMBENS and RUBIN 2015; MERCER et al. 2017).

${ }^{13}$ MERCER et al. (2017: 253) explicitly liken survey research and experimental research: "A probability-based survey is essentially a randomized experiment where the pool of subjects is the set of units on the sampling frame and the treatment is selection into the survey. Unlike experiments where we observe outcomes on both treated and untreated subjects, in surveys we observe outcomes only on the selected units, with the expectation that there should be no difference between selected and non-selected units."

${ }^{14}$ In random sampling, where "data are missing completely at random" (MCAR), unit selection is unconditionally independent of the variable of interest (no confounding). Using propensity scores to "remedy" self-selection, in contrast, is an attempt to make unit selection independent of the variable of interest conditional on observed confounders. This corresponds to the notion of "data missing at random" (MAR). When not all confounders are observed, it is not possible to adequately correct for selection bias. This corresponds to the notion of "data not missing at random" (NMAR) (MERCER et al. (2017). 
participation in real surveys may be much more complex and depend on multiple, and potentially interacting, confounding variables (including the target variable itself) that might furthermore affect participation in a non-linear fashion. Individual participation propensities will therefore often differ between most subjects. As a consequence, simple weighting schemes as the one described above do not suffice any more to account for the biased sample composition introduced by self-selection. More elaborate propensity score models try to accommodate these complexities by considering all potentially relevant characteristics to calculate individual participation probabilities, which, in turn, are considered when estimating population parameters and standard errors. Contrary to the outcome in our stylized example, standard errors that are adjusted for self-selection are often larger than those resulting from the assumption that there is no problem with a biased composition of the sample (COPAS and LI 1997).

While the formal presentation of propensity score models or other sample selection models that deal with the problem of data that are missing but not missing at random is beyond this paper's scope (cf. e.g. LITTLE and RUBIN 2002), it should be recognized that we need to be generally wary of miss-specifying the selection model. When groups with distinct characteristics are completely missing in the sample or when we do not know or are not able to measure all selection-relevant variables, we cannot properly correct for selection bias. While emphasizing that correction methods should be used whenever sufficient data are available, CUDDEBACK et al. (2004: 22) note that "failing to use these methods when appropriate is different than failing to use them when data for non-participants are unavailable, which is common. In this latter case, sample selection models are obviously of no use." Of course, researchers can never be completely sure that they know all selection-relevant variables. And often the necessary data from non-respondents will simply be lacking. But even when considerable amounts of data are available from non-respondents, one should remain skeptical whether all confounding variables that affect selection were adequately considered. Going beyond conventional routines, this needs to be explicitly reflected in the interpretation of results to avoid erroneous inferential reasoning. ${ }^{15}$

We may summarize: in empirical research in the social sciences, we often use convenience samples because we are unable to comply with the "empirical commitment" to randomly sample units from the defined parent population. This is due to two obstacles: first, lacking accessibility to the population or insufficient budgets often prevent researchers from constructing appropriate sampling frames that cover the entire target population. Second, even in appropriate sampling frames, uncontrolled self-selection is likely to produce biased samples. Recent trends in survey research exacerbate these problems: the decade-long trend towards declining survey response (often not more than $20 \%$ or even much less) aggravates the problem of self-selection. In addition, researchers exacerbate the initial problem of inappropriate sampling frames when they increasingly use online survey platforms in response to declining response rates. On such platforms, respondents can be recruited at low costs from non-probability panels of individuals who often volunteer to participate in many different surveys for money. Ignoring how a sample was probabilistically composed from a well-defined population rules out the use of standard errors and $p$-values because no sampling distribution can be envisaged. In some rare cases, we may have

\footnotetext{
${ }^{15}$ Given the trend of decreasing survey participation and increasing competition from alternative data sources, Public Opinion Quarterly published a Special Issue on "Survey Research, Today and Tomorrow" in 2017 (Vol. 81). The Special Issue puts a particular focus on the question of how to deal with non-probability samples. Its editor notes: "To survive, surveys need to maintain their 'value proposition.' One important part of this proposition is that surveys gather information that is generalizable from the measured sample to a well-defined population" (MILLER 2017: 205-207). With a view to the increasing use of non-probability samples obtained from volunteer platforms, he states that " $[\mathrm{t}]$ here have been prominent clashes between advocates for the probability and nonprobability approaches. Those who support the probability sampling method observe that they have probability theory to support their population inferences, while the advocates for volunteer panels rely on inductive modeling that has no comparable theoretical foundation." In response, the supporters of non-probability panels stress that their approaches work well enough in many practical research contexts and counter that actually achieved samples do very rarely come up to the presumed probabilistic requirements anyway.
} 
appropriate sampling frames and enough information from non-respondents to correct for selection bias, which, in turn, rehabilitates the probabilistic foundations for using inferential statistics. But commonly, even when appropriate sampling frames can be provided, we have too little information on sample selection because data from non-respondents are lacking. When we are not able to perform the necessary corrections, we should refrain from delusively insinuating that the composition of the sample is unbiased. Instead, we should realize and communicate that we have to do without inferential statistics (see the above quote by TRAFIMOW 2019). While inductive inferences based on comprehensible scientific arguments are of course feasible in such circumstances, $p$-values are meaningless and cannot be used as inferential aids in the inductive exercise of generalizing from the sample to the parent population.

\subsection{Full population surveys (100\% samples)}

Since statistical inference is concerned with sampling error and generalizations from random samples to parent populations, there is neither need nor room for statistical inference when we already have data for an entire population. VoGT et al. (2014: 243) note that this is quite common, for example, when analyzing aggregated economic data. They also provide an illustrative example of a study that looks at the association between mean education levels and birth rates in all UN member states. Since there is nothing to infer, displaying $p$-values does not make sense. Instead, one should simply describe the population quantities including regression coefficients. This intuitive fact is formally reflected in the finite population correction factor $[(N-n) /(N)]^{0.5}$ : instead of implicitly assuming that a sample was drawn from an infinite population - or at least that a small sample of size $n$ was drawn from a very large population of size $N$ - the finite population correction factor $(f p c)$ considers the fact that, besides absolute sample size, sampling error decreases when the sample size becomes large relative to the whole population (HIRSCHAUER et al. 2019b). The $f p c$ reduces the standard error and should be used when sample share is more than $5 \%$ of the population (KNAUB 2008). Having data for an entire population $(n=N)$ corresponds to a correction factor of zero, which leads a corrected standard error of zero. This is logically consistent because there is no sampling error when the "sample" covers $100 \%$ of the population.

Nonetheless, $p$-values are frequently displayed in regressions that analyze data from entire populations. To justify this procedure, the frequentist statistician must somehow introduce the notion of a sampling distribution and a sampling error. This implies imagining an infinite "unseen parent population" (or "superpopulation") and a generating process from which one has presumably observed one noisy random realization (HIRSCHAUER et al. 2018). In so doing, the observed full population becomes in imagination a sample of a (parent) superpopulation. Even back in the 80s, DENTON (1988: 167) noted that this is a rhetorical device (also known as "great urn of nature") that does not evoke wild enthusiasm from everybody. However, some random process of data generation - and not just a record of empirical data - has to be presumed for frequentist inferential tools such as $p$-values to make sense.

Unfortunately, the presumption of a superpopulation often remains implicit. Sometimes its necessity may even not be realized by researchers themselves who, due to engrained disciplinary habits, engage more or less automatically in "statistical significance testing" whenever running a regression model. Researchers who display $p$-values in the analysis of full-population data should explicitly discuss why and how they base their inferential reasoning on the notion of a superpopulation. This is but a specification of the general desideratum that researchers explicitly describe the population from which the random sample comes and to which they want to generalize.

In some contexts, adopting the perspective of a superpopulation may be comprehensible. Researchers could imagine, for example, a quasi-stable real-world system whose operative regularities, while being possibly limited to a finite time interval, exceed the time interval over which the population data were collected. In the above UN example, this would imply envisaging regularities that not only produced the association between mean education levels and birth rates in all $\mathrm{UN}$ member in the period for which the 
data were collected but that will also be at work in the next period(s). Obviously, the plausibility of such an assumption and thus the validity of generalizing claims supported by $p$-values can only be assessed when the time interval over which the data generating system is presumably at work, is clearly defined.

\section{Conclusion}

The $p$-value is sometimes seen as conclusive piece of evidence. However, in recent years a plethora of publications have warned against inferential errors associated with such views. This includes the $p$-value warning of the American Statistical Association (WASSERSTEIN and LAZAR 2016), a Special Issue in The American Statistician (WASSERSTEIN et al. 2019), and a widely supported p-value petition to retire statistical significance in Nature (AMRHEIN et al. 2019). Following up on these warnings, this paper focused on the often overlooked source of error that the very pre-conditions for using $p$-values are not met when econometric studies are based on non-probability convenience samples. Uncorrected nonprobability samples rule out using $p$-values because inferential statistics are conceptually based on the notion of repeated random sampling (statistical replication) and a resulting sampling distribution. When there is no random sampling, there is no sampling distribution and no random sampling error. Random data generation is therefore a necessary condition for a meaningful application of standard errors and $p$ values. When data do not satisfy this probabilistic requirement, $p$-values are essentially uninterpretable.

Critically reflecting on the conceptual pre-conditions for using $p$-values is important for two reasons: first, it calls to mind the nature of statistical inference, which - even if applicable - solely deals with the uncertainty of estimates caused by random error and which is therefore only a part of scientific inference. Second, it is important because much of econometric research uses data from convenience samples that are not probabilistically obtained from well-defined populations. Convenience samples are affected by one of the two following drawbacks (or both): first, the units on the sampling frame are conveniently chosen but not probabilistically selected from the population (non-coverage). Due to non-coverage, there are unknown differences between the units who are on the sampling frame and those who are not. Second, the units that are on the sampling frame select themselves with unknown probability into the final convenience sample which, if not corrected for, leads to selection bias. While these two features often occur jointly in practical research, each of them suffices to rule out a meaningful use of $p$-values. To ensure successful scientific communication, non-probability samples must therefore be clearly distinguished from probability samples: (i) When researchers succeed in achieving probability samples, they should transparently state from which population the sample was drawn, and consequently, to which population they want to make inductive inferences (generalizations). Without providing such basic information, inference statements are nebulous, at best - and without value, at worst. (ii) When researchers are limited to using non-probability samples that preclude the use of inferential statistics they should be clear about it and refrain from displaying $p$-values. Displaying inferential statistics in circumstances where population inferences cannot be supported by probability theory is likely to cause overconfident inductive conclusions. Alas, it still seems a widespread spontaneous reflex among researchers who often do not explicitly question whether there is a chance model upon which to base statistical inference.

\section{References}

Abadie, A., Athey, S., Imbens, G.W., Wooldridge, J.M. (2017): When should you adjust standard errors for clustering? NBER Working Paper 24003.

Amrhein, V., Greenland, S., McShane, B. (2019): Retire statistical significance. Nature 567: 305-307.

Austin, P.C. (2011): An Introduction to Propensity Score Methods for Reducing the Effects of Confounding in oberservational Studies. Multivariate Behavioral Research 46: 399-424.

Berk, R.A. (1983): An introduction to sample selection bias in sociological data. American Sociological Review, 48(3): 386-398. 
Berk, R.A., Freedman, D.A. (2003): Statistical Assumptions as Empirical Commitments. In: Blomberg, T.G., Cohen, S. (eds.): Law, Punishment, and Social Control: Essays in Honor of Sheldon Messinger ( $2^{\text {nd }}$ ed.). New York, de Gruyter: 235-254.

Berry, D. (2017): A p-Value to Die For. Journal of the American Statistical Association 112(519): 895-897.

Brick, J.M. (2013): Unit Nonresponse and Weighting Adjustments: A Critical Review. Journal of Official Statistics 29(3): 329-53.

Cameron, A.C., Miller, D.L. (2010): Robust inference with clustered data, Working Paper, No. 10-7, University of California, Department of Economics, Davis, CA.

Cameron, A.C., Miller, D.L. (2015): A Practitioner's Guide to Cluster-Robust Inference. Journal of Human Resources 50(2): 279-292.

Cameron, A.C., Trivedi, P.K. (2005): Microeconometrics: Methods and Applications. Cambridge, Cambridge University Press.

Cameron, A.C., Trivedi, P.K. (2009): Microeconometrics using Stata. College Station. Stata Press.

Copas, J.B., Li, H.G. (1997): Inference for Non-random Samples. Journal of the Royal Statistical Society Series B, 59(1): 55-95.

Cuddeback, G., Wilson, E., Orm, J.G., Combs-Orme, T. (2004): Detecting and Statistically Correcting Sample Selection Bias. Journal of Social Service Research 30(3): 19-33.

Denton, F.T. (1988): The significance of significance: Rhetorical aspects of statistical hypothesis testing in economics. In: Klamer, A., McCloskey, D.N., Solow, R.M. (eds.): The consequences of economic rhetoric. Cambridge, Cambridge University Press: 163-193.

de Leeuw, E.D., Hox, J.J., Dillman, D.A. (2008): International handbook of survey methodology. New York, Taylor \& Francis Group.

Friedman, J. (2013): worldbank-blog (https://blogs.worldbank.org/impactevaluations/tools-of-the-trade-when-touse-those-sample-weights).

Gelman, A., Carlin, J. (2017): Some natural solutions to the p-value communication problem-and why they won't work. Journal of the American Statistical Association 112(519): 899-901.

Gigerenzer, G., Marewski J.N. (2015): Surrogate Science: The Idol of a Universal Method for Statistical Inference. Journal of Management 41(2): 421-440.

Greene, W.H. (1981): Sample selection bias as a specification error: Comment. Econometrica 49(3): 795-798.

Greenland, S., Senn, S.J., Rothman, K.J., Carlin, J.B., Poole, C., Goodman, S.N., Altman, D.G. (2016): Statistical tests, $\mathrm{P}$ values, confidence intervals, and power: a guide to misinterpretations. European Journal of Epidemiology 31(4): 337-350.

Guo, S., Fraser, M.W. (2015): Propensity Score Analysis. Statistical Methods and Applications (2 ${ }^{\text {nd }}$ ed.). Thousand Oaks, Sage Publications.

Heckman, J.J. (1976): The common structure of statistical models of truncation, sample selection and limited dependent variables and a simple estimation for such models. Annals of Economic and Social Measurement 5(4): 475-492.

Heckman, J.J. (1979): Sample selection bias as a specification error. Econometrica, 47(1): 153-161.

Heckman, J.J., Ichimura, H., Todd, P.E. (1997): Matching as an econometric evaluation estimator: Evidence from evaluating a job training programme. Review of Economic Studies 64: 605-654.

Hirschauer, N., Grüner, S., Mußhoff, O., Becker, C. (2018): Pitfalls of significance testing and p-value variability: An econometrics perspective. Statistics Surveys 12(2018): 136-172.

Hirschauer, N., Grüner, S., Mußhoff, O., Becker, C. (2019a): Twenty steps towards an adequate inferential interpretation of p-values in econometrics. Journal of Economics and Statistics 239(4): 703-721.

Hirschauer, N., Grüner, S., Mußhoff, O., Becker, C. (2019b): Economic experiments and inference. SocArXiv Papers (Doi: 10.31235/osf.io/67mws).

Imbens, G.W., Rubin, D.B. (2015): Causal Inference for Statistics, Social, and Biomedical Sciences. New York, Cambridge University Press.

Kalton, G., Flores-Cervantes, I. (2003): Weighting Methods. Journal of Official Statistics 19(2): 81-97.

Kish, L. (1965): Survey Sampling. New York, Wiley.

Knaub, J. (2008): Finite Population Correction (fcp) Factor. In: Lavrakas, P. (ed.): Encyclopedia of Survey Research Methods. Thousand Oaks, Sage Publications: 284-286. 
Krämer, W. (2011): The Cult of Statistical Significance - What Economists Should and Should Not Do to Make their Data Talk. Schmollers Jahrbuch 131(3): 455-468.

Lee, B., Marsh, L.C. (2000): Sample selection bias correction for missing response observations. Oxford Bulletin of Economics and Statistics 62(2): 305-323.

Little, R.J.A., Rubin, D.B. (2002): Statistical analysis with missing data (2 ${ }^{\text {nd }}$ ed.). Hoboken, New York, Wiley.

Lohr, S.L. (2009): Sampling: Design and Analysis (2nd ed.). Boston, Brooks/Cole.

Luellen, J.K., Shadish, W.R., Clark, M.H. (2005): Propensity scores: An introduction and experimental test. Evaluation Review 29: 530-558.

Ludwig, D.A. (2005): Use and Misuse of p-Values in Designed and Observational Studies: Guide for Researchers and Reviewers. Aviation, Space, and Environmental Medicine 76(7): 675-680.

MacKinnon, J.G. (2019): How Cluster-Robust Inference is Changing Applied Econometrics. Queen's Economic Department Working Paper no. 1413.

Matthews, R., Wasserstein, R.L., Spiegelhalter, D. (2017): The ASA's p-Value Statement, One Year on. Significance 14: 38-41.

McCloskey, D.N., Ziliak, S.T. (1996): The Standard Error of Regressions. Journal of Economic Literature 34(1): 97-114.

Mercer, A.W., Kreuter, F., Keeter, S., Stuart, E. (2017): Theory and Practice in Nonprobability Surveys. Parallels between Causal Inference and Survey Inference. Special Issue 2017: Survey Research, Today and Tomorrow, Public Opinion Quarterly 81: 250-279.

Miller, P.V. (2017): Is there a Future for Surveys? Editorial to the Special Issue 2017: Survey Research, Today and Tomorrow, Public Opinion Quarterly 81: 205-212.

Rosenbaum, P.R. (2010): Design of observational studies. New York, Springer.

Rosenthal, R., Rosnow, R.L. (2009): Artifacts in Behavioral Research. Oxford, Oxford University Press.

Shadish, W.R., Cook, T.D., Campbell, D.T. (2002): Experimental and quasi- experimental designs for generalized causal inference. Boston, Houghton Mifflin.

Solon. G., Haider, S.J., Wooldridge, J. (2013): What are we weighting for? NBER Working Paper 18859 (http://www.nber.org/papers/w18859).

Stolzenberg, R.M., Relles, D.A. (1997): Tools for intuition about sample selection bias and its correction. American Sociological Review 62(3): 494-507.

Trafimow, D. et al. (2018): Manipulating the alpha level cannot cure significance testing. Frontiers in Psychology 9 (https://www.frontiersin.org/articles/10.3389/fpsyg.2018.00699/full).

Trafimow, D. (2019): Five Nonobvious Changes in Editorial Practice for Editors and Reviewers to Consider When Evaluating Submissions in a Post p < 0.05 Universe. The American Statistician, 73(sup1): 340-345.

Valliant, R. Dever, J.A., Kreuter. F. (2013): Practical Tools for Designing and Weighting Survey Samples. New York, Springer.

Vella, F., Verbeek, M. (1999): Two-step estimation of panel data models with censored endogenous variables and selection bias. Journal of Econometrics 90(2): 239-263.

Vogt, W.P., Vogt, E.R., Gardner, D.C., Haeffele, L.M. (2014): Selecting the right analyses for your data: quantitative, qualitative, and mixed methods. New York, The Guilford Publishing.

Winship, C., Mare, R.D. (1992): Models for sample selection bias. Annual Review of Sociology 18: 327-350.

Wasserstein, R.L., Lazar N.A. (2016): The ASA's statement on p-values: context, process, and purpose, The American Statistician 70(2): 129-133.

Wasserstein, R.L., Schirm, A.L., Lazar, N.A. (2019): Moving to a World Beyond “p < 0.05”. The American Statistician 73(sup1): 1-19.

Ziliak, S.T., McCloskey D.N. (2008): The Cult of Statistical Significance. How the Standard Error Costs Us Jobs, Justice, and Lives. Michigan, The University of Michigan Press. 